\title{
Prevalence and Severity of Temporomandibular Disorders in Medical/Dental Undergraduate Students
}

\author{
Mahvish Wahad Khan ${ }^{1}$ \\ BDS \\ Abdul Mueed Zaigham² \\ BDS, FCPS
}

OBJECTIVE: The objective of this epidemiological study was to assess the prevalence and severity of temporomandibular disorders (TMD) in undergraduate students at CMH Lahore Medical College (CMH-LMC) and Institute of Dentistry (IOD) using Fonseca's questionnaire.

METHODOLOGY: A cross-sectional survey study was conducted. A questionnaire was distributed among 644 undergraduate students attending CMH Lahore Medical College (CMH-LMC) and Institute of Dentistry (IOD). The questionnaire had some general questions regarding medical and dental history requiring responses in yes or no and it had 10 specific questions regarding the temporomandibular joint with response options yes, sometimes \& no which were allocated scores 10, 5 and 0 respectively. The sum of points was used to classify participants into TMD free, mild, moderate, and severe temporomandibular disorders (TMD).

RESULTS: The prevalence of TMD was found to be $62 \%$. Among all the participants almost 37\% were TMD free, $43 \%$ students suffered from Mild TMD, 18\% students had moderate TMD and 1\% students suffered from severe TMD. CONCLUSION: The Fonseca's questionnaire can be used as an effective tool in assessment of prevalence and severity of temporomandibular disorders. Public health services should adopt the questionnaire for screening, as information can be obtained in a relatively short period and at a low cost for a wide population.

KEYWORDS: Fonseca's questionnaire, Temporomandibular disorders, Orofacial pain, Cervicofacial muscles.

HOW TO CITE: Khan MW, Zaigham AM. Prevalence and severity of temporomandibular disorders in medical/dental undergraduate students. J Pak Dent Assoc 2021;30(2):94-98.

DOI: https://doi.org/10.25301/JPDA.302.94

Received: 18 October 2020, Accepted: 29 January 2021

\section{INTRODUCTION}

$\mathrm{T}$ emporomandibular joints (TMJ) are hinge joints which connect the jaw bone to the skull and are responsible for the complex jaw movements. Any alterations in these joints and associated structures can affect normal functioning of an individual. The term "Temporomandibular Disorders" (TMD) collectively narrates a subgroup of orofacial disorders. These disorders are characterized by pain, limitation of mandibular movements, fatigue to cranio cervicofacial muscles especially the masticatory muscles and presence of articular clicking. ${ }^{1,2,3,4}$ The causes and therapeutic treatment for these disorders have long been an area of interest. ${ }^{1}$

The temporomandibular disorders have a multifactorial etiology. Psychological, biomechanical, biological and neuromuscular factors may contribute to the disorder. ${ }^{5}$ An

1. Post Graduate Resident, Department of Prosthodontics, Institute of Dentistry, CMH Lahore Medical College, Lahore, National University of Medical Sciences (NUMS).

2. Professor, Associate Dean \& Head of Prosthodontics department, Institute of Dentistry, CMH Lahore Medical College, Lahore (NUMS).

Corresponding author: "Dr. Mahvish Wahad Khan" < docmahvish@gmail.com > understanding of the etiology of TMD is essential for identifying and avoiding probable pathological factors. The causative psychological factors may include emotional stress, anxiety and depression. Malalignment or loss of teeth, grinding and clenching of teeth, non-functional movements of mandible (bruxism) and occlusal interferences could be the biomechanical causative factors. . $^{1,2,3,4,5}$ Increased level of estrogen hormone has been identified as a biological factor affecting TMJ. Poor posture, defective functioning of masticatory muscles \& adjacent structures, changes within $\&$ around the joint, traumatic injuries, neoplastic growths, immune-mediated systemic diseases and hereditary factors may also contribute to the disease. ${ }^{1,2,3,4} \mathrm{An}$ amalgamation of the above-listed factors can also lead to TMD. ${ }^{1,2,3,4}$ Prosthodontic rehabilitation, orthodontic intervention through non-surgical or surgical means and fractures of jawbone have been perceived to alter or worsen the existing conditions. ${ }^{2,3}$ The etiological factors of TMD can also be classified into predisposing factors which increase the risk of developing TMD, initiating factors which cause disease onset and perpetuating factors which enhance disease propagation or interfere with the healing. ${ }^{5}$ 
Prevalence and severity of temporomandibular disorders in medical/dental undergraduate students

The prevalence of TMD varies from $20 \%$ to $50 \%$. This variation may be attributed to different racial origins, sampling techniques and diagnostic criteria used. ${ }^{2}$ The TMD have been reported to be three times more prevalent in females compared to males. ${ }^{3,4}$ According to a study done on university students, TMD and its associated symptoms were found to be frequent among students of health and science studies. Females showed a greater prevalence of signs and symptoms of TMD than males. ${ }^{6}$ In another study a positive correlation of TMD diagnosis existed with psychological parameters as well as functional occlusal parameters among university students. ${ }^{7}$ TMDs were also frequently diagnosed in a group of Polish young adults. ${ }^{8}$

Screening of TMD in a population is challenging. Various instruments for the screening have been proposed however, no universal diagnostic criteria is not yet available. ${ }^{1,2} \mathrm{TMD}$ are progressive and tend to worsen with time. Therefore, an early diagnosis and treatment are of great value. ${ }^{1,3,4} \mathrm{~A}$ selfadministered questionnaire comprising of Fonseca's anamnestic index ( Da Fonseca et al 1994) initially developed for Brazilian Portuguese population has been put forward as a simple, cost-effective and handy tool for assessing the severity of TMD based on signs and symptoms. ${ }^{1,2,9}$ It has proven to be reliable and provides valuable information about disease severity without much influence from interviewer and variation in assessment of disease status. ${ }^{1,2,9,10}$

This study will help to identify cases of TMD among the undergraduate medical/dental students at an early stage and its causative factors contributing to progression of the disease will be highlighted for early treatment of individuals. Identification of mild to moderate cases will help to prevent their progression to severe forms of disease which could be debilitating.

\section{METHODOLOGY}

A cross-sectional survey was conducted in CMH-LMC and IOD, using the questionnaire proposed by Fonseca for identifying TMD and classifying its severity (Table -1). ${ }^{1,2,4,9}$ The study was approved by ethical review committee of the institution (Case \#. 391/ERC/CMH/LMC). The Fonseca's questionnaire was composed of ten questions, checking for any difficulty in mouth opening, presence or absence of pain during side-to-side jaw movements or chewing, complain of frequent headaches or earaches, pain or stiffness of the neck, parafunctional habits, joint clicking, feeling of improper contact between teeth and perception of emotional stress.

The participants were briefed that the questions need to be answered with "yes" (10 points), or "no" (0 points), or "sometimes" (5 points) and for each question one best answer has to be selected. The sum of the points scored by the
Table - 1: Fonseca's Questionnaire

\begin{tabular}{|l|l|}
\hline Sr. No. & Question responses: Yes, Sometimes and No. \\
\hline 1. & Is it hard for you to open your mouth? \\
\hline 2. & Is it hard for you to move your mandible (jaw)from side to side? \\
\hline 3. & Do you get tired /muscular pain while chewing? \\
\hline 4. & Do you have frequent headaches? \\
\hline 5. & Do you have pain on the nape or stiff neck? \\
\hline 6. & Do you have earaches or pain in craniomandibular joints? \\
\hline 7. & $\begin{array}{l}\text { Have you noticed any TMJ clicking while chewing or when you open your } \\
\text { mouth? }\end{array}$ \\
\hline 8. & Do you clench or grind your teeth? \\
\hline 9. & Do you feel your teeth do not articulate well? \\
\hline 10. & Do you consider yourself a nervous (tense) person? \\
\hline
\end{tabular}

participants was utilized for classifying them into the following four categories: A score of $0-15$ as TMD free, a score of 20-40 as mild TMD, a score of 45-65 as moderate TMD and a score of 70-100 as severe TMD.

Full time medical and dental undergraduate students in CMH-LMC and IOD, who consented for participation in the study and had no history of orthodontic treatment were included in the study. However, those with a history of trauma to the temporomandibular joint, any systemic illness, musculoskeletal or neurological disorders, and those undergoing orthodontic treatment or taking regular analgesic or antianxiety medications were excluded from the study. The questionnaire was distributed among the six hundred and fourty-four students. The students were briefed about filling the questionnaire.

The collected data was entered in Statistical Package for Social Sciences (SPSS) version \# 22. For the quantitative variables mean and standard deviation were calculated whereas for qualitative variables frequencies and percentages were generated. To find out any significant association/relationship between TMD and age, gender and year of student's study Chi-Square test was applied. A p-value of $<0.05$ was considered significant.

\section{RESULTS}

The number of students studying in the institution at the time of the study was 859 , two hundred and fifteen students did not give consent to be part of the study and one hundred and ninety-four students were excluded based on exclusion criteria. The response rate was $75 \%$. A total of four hundred and fifty students were included in the study, two hundred and eighty-five females and one hundred and sixty-five males. Their age ranged from seventeen to twenty-seven years. The mean age of respondents was almost twenty-one 
years with 1.6 standard deviation. Among the total number of students, one hundred and fifteen students studied in the first year, one hundred and seventeen students in the second year, eighty in the third year, eighty-six in the fourth year and fifty-two students were in the fifth year.

One hundred and sixty-seven $(37.11 \%)$ students were TMD free, while one hundred and ninety-four $(43.11 \%)$ had mild TMD, eighty-three $(18.44 \%)$ had moderate and six $(1.33 \%)$ had severe TMD. Almost two-third, $63 \%$ of students had some form of TMD. (Figure - 1)

Figure - 1: Frequency of TMD among study participants

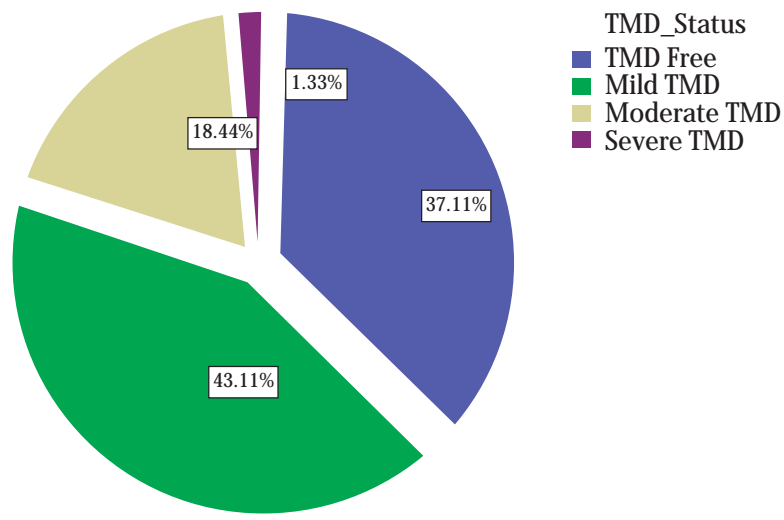

Among the participants, two hundred and seven (46\%) were aged between 17-21 and two hundred and fourty-three (54\%) were aged above 22 years. More participants of the younger age group were TMD free as compared to the older group. All three categories of TMD were found more in older students than younger ones. (Figure -2)

Figure - 2: Association of TMD with age
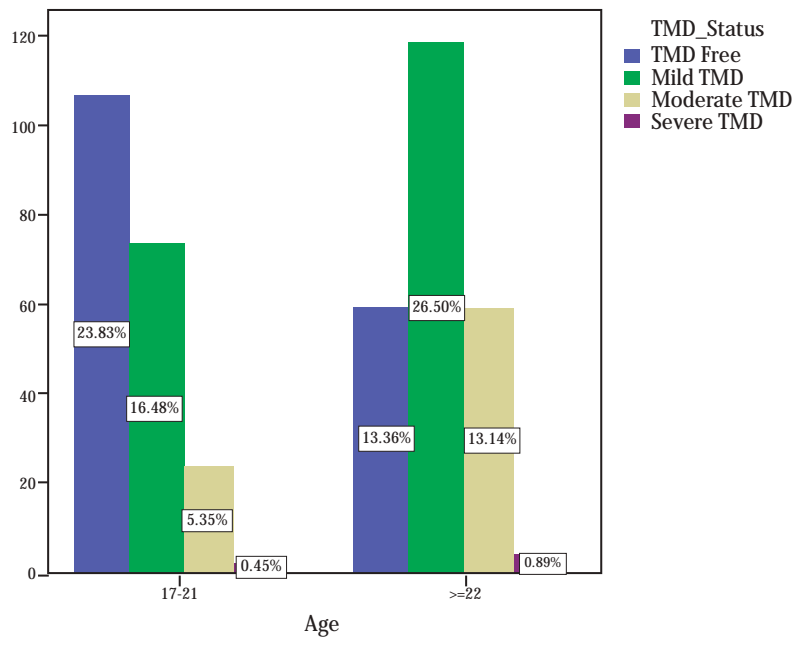

Fifteen percent (15\%) males suffered from mild TMD whereas $28 \%$ females suffered from mild TMD. Moderate
TMD was also observed more in females whereas severe TMD though had low prevalence was reported more in males. (Figure - 3)

Figure - 3: Association of TMD with gender

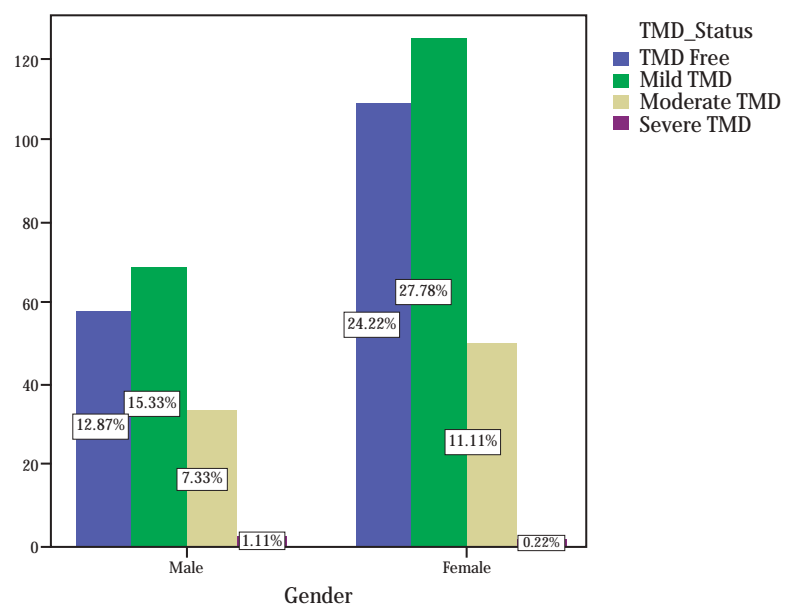

The number of TMD free cases gradually declined from the first year to the final year of study. The prevalence of mild TMD was highest in the fourth-year students. Moderate TMD had the highest prevalence among the third-year students. Severe TMD though had a low prevalence with most cases $(2 \%)$ in the second year. The significant p-value (0.000) demonstrated that TMD status was found to be different among different study years. (Figure - 4)

Figure - 4: TMD percenatge by study year TMD percentage by study year

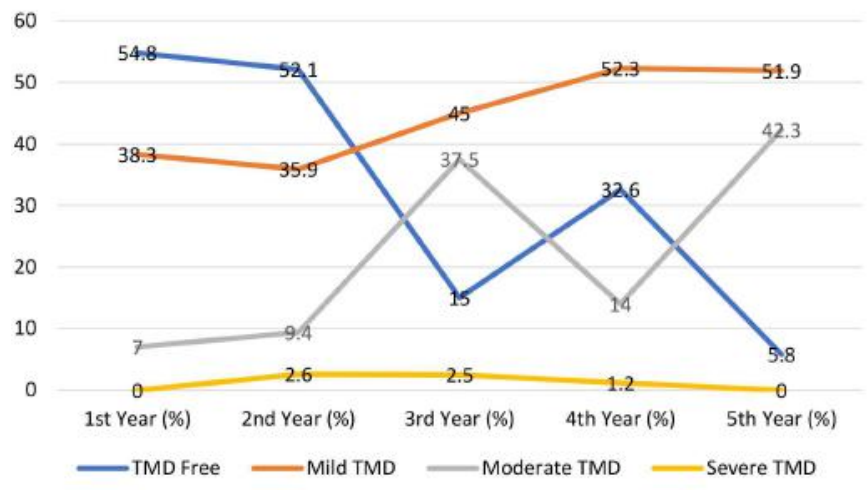

\section{DISCUSSION}

The study equips about the presence and extent of TMD among undergraduate students in CMH-LMC and IOD, established on the basis of Fonseca's questionnaire. The causative and contributory factors of TMD are highly variable, necessitating screening in different populations. Fonseca's 
questionnaire is self-administered, making it convenient to collect information without the influence of the researcher. In some previous studies (Dworkin and Lersche ${ }^{11} 1992$, Schiffman and colleagues ${ }^{12}$ 2014) different methods and parameters have been used to assess TMD including physical examination and radiographic interpretation as a tool whereas others relied only on a questionnaire.

A variation in the prevalence of TMD based on Fonseca's criteria has been noted in previously published studies. In the current study, the prevalence was $63 \%$. This prevalence rate was quite similar to FAI-based TMD prevalence rates (42-68\%) reported by other researchers. ${ }^{2,3,13,14,15}$

Wahid et al. showed $92 \%$ prevalence of TMD, in his study all participants were females. ${ }^{4}$ The variation in the prevalence of TMDs can be attributed to ethnic background, sample size and gender-related variations. The estimated prevalence of disease could be lower than actual since $25 \%$ of students did not consent to participate in the study. Two hundred and thirty participants reported to be suffering from psychological stress and the high prevalence rate can be attributed to their stressful mental state. Psychological stress is a known causative factor for TMD. ${ }^{1,2,3,4,5}$ A positive association between TMD and stress was also observed in another recent study. ${ }^{7}$ Stress in medical/ dental students is usually related to burden of studies and fulfilment of minimal clinical requirements specified for each graduate year.

A mild form of the disease had the highest prevalence in our study, a similar prevalence was found in a study perfomed by Karthik et $\mathrm{al}^{6}$ and similar studies by Pedroni et $\mathrm{al}^{16}$ and Dekon et al. ${ }^{17}$

In the current study, the elder age group ( $>22$ years) showed more prevalence and severity of TMDs as compared to the younger age group. Few other studies also reported an increase in symptoms with increasing age. ${ }^{3,18}$ This association of disease with age can be linked to increase in responsibilities and social pressures related to studies, performance and jobs as well as to self-reported health concerns. Few previous studies did not link TMD with age. ${ }^{1,2}$

The present study showed higher TMD levels in female students than in males, which was consistent with the results of some previous studies. ${ }^{3,6,14,19}$ Both mild and moderate forms of TMD were observed more in female students. The female predominance could be attributed to higher stress levels in the female students, their contrasting physiological characteristics including hormonal variations, different muscle structure and discrete genetic makeup. ${ }^{14,20}$ Studies in Western societies showed an equal distribution of TMD among males and females. ${ }^{21,22}$ Whereas some other studies reported in the literature were entirely conducted on males ${ }^{2}$ or females ${ }^{4,13,14,19,23}$ or the study population did not allow any gender comparison.
TMD prevalence was also compared between the various study years. It was observed that TMD free cases gradually declined from the first year to the second year whereas they significantly declined in the third year followed by an incline in the fourth year and again a significant decline in the fifthyear students. Mild TMD cases showed a more or less constant incline from the first year to the fifth year. Moderate cases showed a significant incline in third and fifth years. Severe TMD was non-existent in first and fifth years while varied between $1-3 \%$ in the rest of the three years. These observations could be attributed to the increase in stress and pressure at the start of clinical rotations in the third year of undergraduate studies which continues till the final year with the family and social pressure increasing as everyone is looking forward to see them as the young fresh graduates. No such comparison was reported in previous studies.

\section{CONCLUSIONS}

From this study, it was concluded that a simple amnestic index is useful in identifying and classifying TMD symptoms according to severity. More than half of the medical/dental students suffer from various degrees of TMD. Mild TMD was most prevalent TMD form in the study sample. Moderate TMD was most significant in third-year students which could be related to the stress of clinical rotations. Prevalence of disease increases with age and females suffer more from TMD than males.

\section{LIMITATIONS}

The study used a brief questionnaire, convenience sampling and included only medical and dental undergraduates. Clinical and radiographic evaluation of the study population was not done and biological factors (hormone levels) were not assessed. More studies should be conducted to corelate clinical and radiographic findings with the findings from the questionnaire so that awareness can be created among the individuals and comprehensive treatment can be provided.

\section{CONFLICT OF INTEREST}

None to declare

\section{REFERENCES}

1. Chandak RM, Pandhripande RM, Sonule SS, Chandak MG, Rawlani SS. To assess the prevalence of signs and symptoms of temporomandibular disorders in Vidarbha population by Fonseca's questionnaire. J Oral Res Rev 2017;9:62-6.

https://doi.org/10.4103/jorr.jorr_7_17 
2. Habib SR, Al Rifaiy MQ, Awan KH, Alsaif A, Alshalan A, Altokais Y. Prevalence and severity of temporomandibular disorders among university students in Riyadh. Saudi Dent J. 2015;27:125-30. https://doi.org/10.1016/j.sdentj.2014.11.009

3. Zareef U, Arshad S, Abid M, Qureshi NR, Ali SA (2018) Frequency of Temporomandibular Joint Disorders among Medical and Dental Undergraduate students in Karachi by Fonseca Questionnaire. J Oral Hyg Health 6:242;2332-702

https://doi.org/10.4172/2332-0702.1000242

4. Wahid A, Mian FI, Razzaq A, Bokhari SAH, Kaukab T, Iftikhar A, Khan H. Prevalence and Severity of Temporomandibular Disorders (TMD) in undergraduate medical students using Fonseca's Questionnaire. Pak Oral Dent J Vol 34, 2014:38-41

5. Chisnoiu AM, Picos AM, Popa S, Chisnoiu PD, Lascu L, Picos A, Chisnoiu R. Factors involved in the etiology of temporomandibular disorders-a literature review. Clujul Med. 2015;88:473. https://doi.org/10.15386/cjmed-485

6. Karthik R, Hafila MF, Saravanan C, Vivek N, Priyadarsini P, Ashwath B. Assessing prevalence of temporomandibular disorders among university students: a questionnaire study. J Int Soc Prevent Community Dent. 2017;7(Suppl 1):S24.

https://doi.org/10.4103/jispcd.JISPCD_146_17

7. Jivnani HM, Tripathi S, Shanker R, Singh BP, Agrawal KK, Singhal $\mathrm{R}$. A study to determine the prevalence of temporomandibular disorders in a young adult population and its association with psychological and functional occlusal parameters. J Prosthod. 2019;28:e445-e449. https://doi.org/10.1111/jopr.12704

8. Loster JE, Osiewicz MA, Groch M, Ryniewicz W, Wieczorek A. The prevalence of TMD in Polish young adults. J Prosthod. 2017;26:284-8

https://doi.org/10.1111/jopr.12414

9. Berni, K.C.S., et al., Accuracy of the Fonseca anamnestic index in the identi?cation of myogenous temporomandibular disorder in female community cases, J Bodywork Movement Therap (2014) http://dx.doi.org/10.1016/j.jbmt.2014.08.001

10. Campos JA, Carrascosa AC, Bonafé FS, Maroco J. Severity of temporomandibular disorders in women: validity and reliability of the Fonseca Anamnestic Index. Brazilian Oral Res. 2014;28:1-6. https://doi.org/10.1590/1807-3107BOR-2014.vol28.0033

11. Truelove EL, Sommers EE, LeResche L, Dworkin SF, Von Korff M. Clinical diagnostic criteria for TMD new classification permits multiple diagnoses. J Am Dent Assoc. 1992;123:47-54. https://doi.org/10.14219/jada.archive.1992.0094

12. Peck CC, Goulet JP, Lobbezoo F, Schiffman EL, Alstergren P, Anderson GC, de Leeuw R, Jensen R, Michelotti A, Ohrbach R, Petersson A. Expanding the taxonomy of the diagnostic criteria for temporomandibular disorders. J Oral rehabilitation. 2014 ;41:2-3. https://doi.org/10.1111/joor.12132
13. Modi P, Shaikh SS, Munde A. A cross sectional study of prevalence of temporomandibular disorders in university students. Int J Sci Res Publ. 2012;2:1-3.

14. Nomura K, Vitti M, Oliveira AS, Chaves TC, Semprini M, Siéssere S, Hallak JE, Regalo SC. Use of the Fonseca's questionnaire to assess the prevalence and severity of temporomandibular disorders in Brazilian dental undergraduates. Brazi Dent J. 2007;18:163-67.

https://doi.org/10.1590/S0103-64402007000200015

15. Oliveira AS, Dias EM, Contato RG, Berzin F. Prevalence study of signs and symptoms of temporomandibular disorder in Brazilian college students. Brazi Oral Res. 2006;20:3-7.

https://doi.org/10.1590/S1806-83242006000100002

16. Pedroni CR, De Oliveira AS, Guaratini MI. Prevalence study of signs and symptoms of temporomandibular disorders in university students. J Oral Rehabil. 2003;30:283-9.

https://doi.org/10.1046/j.1365-2842.2003.01010.x

17. Dekon SF, Fajardo RS, Zavanelli AC, Beleeiro RP, Pelisser J. Estudo. Comparative study between anamnestic index of TMD and inventory Of trait-state anxiety (IDATE) JBA J Bras ATM occlusion Dor Orofac. 2002;2:224-7.

18. Gillborg S, Åkerman S, Lundegren N, Ekberg EC. Temporomandibular Disorder Pain and Related Factors in an Adult Population: A Cross-Sectional Study in Southern Sweden. J Oral Fac Pain Headache. 2017;31:37-45. https://doi.org/10.11607/ofph.1517

19. Ashfaq M, Bangash TH, Munim A. Associated features of temporomandibular pain dysfunction syndrome. J Postgraduate Med Insti Peshawar. 2007;21:178-82.

20. Mottaghi A, Razavi SM, Pozveh EZ, Jahangirmoghaddam M. Assessment of the relationship between stress and temporomandibular joint disorder in female students before university entrance exam (Konkour exam). Dent Res J. 2011;8(Suppl1):S76.

21. Bevilaqua-Grossi D, Chaves TC, De Oliveira AS, Monteiro-Pedro V. Anamnestic index severity and signs and symptoms of TMD. CRANIOß. 2006;24:112-8.

https://doi.org/10.1179/crn.2006.018

22. Celic R, Jerolimov V, Zlataric DK. Relationship of slightly limited mandibular movements to temporomandibular disorders. Brazi Dent J. 2004;15:151-4. https://doi.org/10.1590/S0103-64402004000200012

23. Bonini JA, Campos DD, Carrascosa AC, Bonafé FS, Maroco J. Epidemiology of severity of temporomandibular disorders in Brazilian women. J Oral Fac Pain Headache. 2014;28:147-52 